\title{
Nucleobase Protection of Deoxyribo- and Ribonucleosides
}

\section{SALIENT FEATURES OF OLIGONUCLEOTIDE SYNTHESIS}

DNA and RNA are biopolymers comprised of nucleotide units. The synthesis of DNA and RNA as segments consisting of several nucleotides each, referred to as oligonucleotides, is quite a challenging endeavor, requiring the rapid and quantitative coupling of nucleosidic units to form internucleotidic linkages. In order to achieve high efficiency in the coupling reactions, it is necessary to direct the reaction to the desired site in the nucleoside. This is achieved by using appropriate protecting group strategies.

To date, five methods of oligonucleotide synthesis have been reported, all of which use protected nucleosides: (1) The $H$-phosphonate approach (Michelson and Todd, 1955; Hall et al., 1957; Froehler et al., 1986, and references therein; Garegg et al., 1986, and references therein; UNIT 3.4); (2) the phosphodiester approach, pioneered by Khorana and co-workers (Gilham and Khorana, 1958; Khorana, 1979); (3) the phosphotriester approach, which heralded a new era in nucleic acid synthesis by enabling more rapid synthesis and purification (Letsinger and Ogilvie, 1969, and references therein; Reese, 1978); (4) the phosphite strategy (Letsinger and Lunsford, 1976, and references therein; Matteucci and Caruthers, 1980); and (5) the phosphoramidite approach, presently the most popular method for oligonucleotide synthesis (Beaucage and Caruthers, 1981; UNIT 3.3). Over the years, improvements in coupling chemistries, along with advances in methods of solid-phase assembly, have revolutionized the art of oligonucleotide synthesis.

In the synthesis of oligonucleotides, persistent as well as transient protection of amino, imido, and hydroxy groups of nucleosides are employed. Transient protecting groups are used to temporarily block one of the hydroxy groups. For example, the 4,4'-dimethoxy trityl group (DMTr) is used for transient protection of the $5^{\prime}$ hydroxyl of a nucleoside (UNIT 2.3). The transient protecting group is removed at the beginning of each coupling cycle. This enables the coupling reaction to be directed to the desired hydroxy group during the synthesis cycle. On the other hand, persistent protecting groups are those that remain on the nucleoside throughout chain assembly and are only removed at the end of synthesis. For example, the exocyclic amino groups of nucleobases, the 2'-hydroxy groups of sugars (UNIT 2.2), and the internucleotidic phosphodiester functions, are protected using persistent protecting groups.

The present commentary addresses issues related to the persistent protection of nucleobases. The commentary is not a comprehensive review of published literature but is intended to highlight the salient features of nucleobase protection. Only selected references are cited, and readers should refer to recent reviews (Beaucage and Iyer, 1992; Iyer and Beaucage, 1999) and primary literature for more comprehensive citations.

\section{GENERAL ASPECTS OF NUCLEOBASE PROTECTION}

The choice of nucleobase protecting groups and deprotection protocols is of paramount importance in oligonucleotide synthesis, and is dictated by many considerations. The protecting groups for nucleosides should be designed on the basis of several criteria, which have been summarized by Reese (1978). (1) It should be possible to introduce the protecting group using a stable reagent that is readily obtainable. (2) The group should be achiral. (3) The nuclear magnetic resonance (NMR) spectrum of the protected nucleoside should be simple to interpret. (4) The protecting group should be readily installed on the nucleoside. (5) The group should enhance the solubility of the nucleoside in organic solvents so that it can be adapted for coupling reactions. (6) The group should be stable to reagents employed in oligonucleotide synthesis. (7) The group should not cause other structural changes in the nucleoside during its installation or removal, or during oligonucleotide assembly.

A number of nucleobase protecting groups have been reported. Improved procedures for their installation and removal have been published. Nevertheless, unforeseen problems have been reported when certain protecting groups are employed in oligonucleotide synthesis. For example, $N$-acylated purine nucleosides, particularly $N$-acylated deoxyadenosine, are more predisposed to depurination than their unprotected counterparts, making the growing oligonucleotide chain susceptible to cleavage during the synthesis cycle. In fact, each pro-
Protection of Nucleosides for Oligonucleotide Synthesis 
tected purine and pyrimidine nucleobase exhibits different patterns of reactivity compared with the unprotected nucleoside. Certain protecting groups are also sensitive to reagents used in oligonucleotide synthesis, resulting either in their premature removal or in production of base-modified products (reviewed by Beaucage and Iyer, 1992).

The sections that follow attempt to highlight the structural basis for the nucleophilicity and reactivity of nucleosides. An overview of the strategies employed for nucleobase protection is also provided.

\section{NUCLEOSIDE TAUTOMERISM AND pKa VALUES}

The nucleophilicity of nucleobases (Fig. 2.1.1) is dictated by the $\mathrm{pK}_{\mathrm{a}}$ of the amino and amido functions and their tautomeric forms. Table 2.1.1 lists the $\mathrm{pK}_{\mathrm{a}}$ values of nucleobases. The amide-like nitrogens (N3 of uridine and $\mathrm{N} 1$ of guanosine) are acidic in character, whereas the ring nitrogens are basic. Therefore, at strongly alkaline $\mathrm{pH}$, the proton at $\mathrm{N} 3$ of uridine and thymidine and that at $\mathrm{N} 1$ of guanosine are removed. Under acidic conditions (at $\mathrm{pH} \sim 3$ ), the sites of protonation are N1 of adenosine and N3 of cytidine. At more acidic $\mathrm{pH}$, the $\mathrm{N} 7$ of guanosine and adenosine and $\mathrm{O} 4$ of uridine are protonated. Thus, all the bases remain mostly uncharged in the physiological range of pH 5 to 9 (Saenger, 1984).

It is noteworthy that each of the nucleosides A, C, and $\mathrm{G}$ becomes protonated at one of the ring nitrogens rather than on the exocyclic amino group. Thus, the electron pair of the amino group can delocalize into the heteroaromatic ring. Indeed the $\mathrm{C}-\mathrm{NH}_{2}$ bonds of $\mathrm{A}, \mathrm{C}$, and $\mathrm{G}$ are $\sim 1.34 \AA$ long and have $40 \%$ to $50 \%$ double-bond character (Saenger, 1984). The charge on a nucleobase, its tautomeric structures, and its ability to form and accept hydrogen bonds are also determined by its $\mathrm{pK}_{\mathrm{a}}$ values as well as by the $\mathrm{pH}$ of the medium. At physiological $\mathrm{pH}$, the major nucleobases exist almost exclusively in the amino and keto tautomeric forms. However, under appropriate $\mathrm{pH}$ condi-
Table 2.1.1 $\mathrm{pK}_{\mathrm{a}}$ Values of Nucleoside Nucleobases

\begin{tabular}{ll}
\hline $\begin{array}{l}\text { Nucleobase (site of protonation/ } \\
\text { deprotonation) }\end{array}$ & $\mathrm{pK}_{\mathrm{a}}$ \\
\hline 2'-Deoxythymidine (N3) $^{\prime}$ - & 9.93 \\
Guanosine (N1) & 9.42 \\
Uridine (N3) & 9.38 \\
Cytidine (N3) & 4.17 \\
Adenosine (N1) & 3.52 \\
\hline${ }^{a}$ For numbering of nucleoside positions, see Figure 2.1.1. \\
For additional pK ${ }_{\mathrm{a}}$ values, see Clauwaert and Stockx \\
(1986); Dunn and Hall (1975).
\end{tabular}

tions, the tautomeric equilibrium could shift to where both imino and enol forms could exist (Saenger, 1984). In turn, these factors influence the reactivity of the nucleosides.

\section{REACTIVITY OF NUCLEOSIDES}

Nucleosides participate in electrophilic and nucleophilic substitution reactions as well as addition reactions (Shabarova and Bogdanov, 1994). Quite clearly, protecting groups and the protocols for their installation and removal should be designed to avoid various side reactions.

Nucleobases undergo substitution reactions with electrophilic reagents. For example, both $\mathrm{N}$ - and $\mathrm{O}$-alkylation of the imide and lactam groups occur with alkylating agents. The N7 position of purines is also a potential site for electrophilic attack (Fig. 2.1.5). Because of these competing reactions, simple alkylation of exocyclic amino function is not a viable protection strategy for nucleobases. On the other hand, it is possible to chemoselectively acylate the exocyclic amino group. Thus, acyl-type protecting groups are widely used for the protection of the exocyclic amino groups of nucleosides (Fig. 2.1.7).

The imide/lactam $\mathrm{NH}$ of thymidine, uridine $\left(\mathrm{pK}_{\mathrm{a}}, 9.38\right)$, and guanosine $\left(\mathrm{pK}_{\mathrm{a}}, 9.42\right)$ is weakly acidic and can deprotonate under basic conditions. The resulting nucleophilic anion can react with a variety of reagents such as activated phosphates, dicyclohexylcarbodi-
Nucleobase Protection of Deoxyribo- and Ribonucleosides

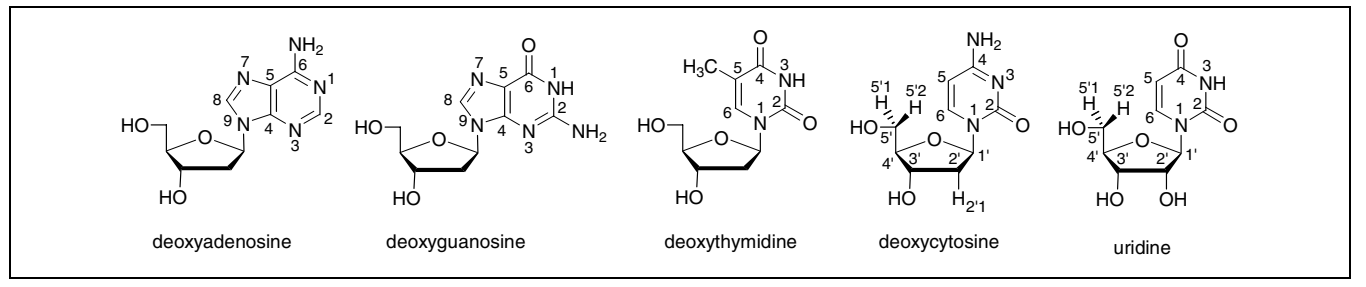

Figure 2.1.1 Structures and numbering of nucleosides. 
imide (DCC), mesitylene sulfonyl chloride, 1(mesitylene-2-sulfonyl)-3-nitro-1,2,4-triazole (MSNT), acid chlorides, phosphitylating reagents, and electrophilic reagents that are employed during coupling reactions. These side reactions result in nucleobase-derived $\mathrm{N}$ - and O-products.

Nucleosides also react with a variety of nucleophilic reagents. For example, cytosine reacts with hydroxylamine at neutral and acidic $\mathrm{pH}$ to give the corresponding hydroxylamine derivative. It reacts with hydrazine at neutral $\mathrm{pH}$ to give the corresponding hydrazide. Indeed, nucleophilic substitution reaction of the exocyclic amino groups of cytosine proceeds rapidly in the presence of alkali and amines.

Under oxidative conditions, certain nucleobases (for example, adenine and cytosine) can form $N$-oxides. Also, the $\mathrm{C} 8$ position of guanosine is vulnerable to hydrolytic attack under either strongly acidic or strongly alkaline conditions. The 5,6 double bond of pyrimidine nucleosides also reacts with halogens and halohydrins to give the corresponding addition products (Shabarova and Bogdanov, 1994). Selected examples of the side reactions that occur during oligonucleotide synthesis are given below.
Tri- $O$-acetyluridine (S.1) reacts with MSNT to produce the triazolo derivative S.2 (Fig. 2.1.2; Reese and Ubasawa, 1980). During deprotection with ammonium hydroxide, $\mathbf{S . 2}$ gives cytidine (S.3). Interestingly, during deprotection with either the tetramethylguanidinium salt of syn-4-nitrobenzaldoxime or tetrabutylammonium fluoride (TBAF), S.2 reverts to uridine (den Hartog et al., 1982).

The triazolo derivative $\mathbf{S . 2}$ is also known to react with other nucleophiles. For example, in the presence of methanol and 1,8-diazabicyclo[3.4.0] undecene-7 (DBU), S.2 gives the corresponding 4-OMe derivative ( $\mathrm{Li}$ et al., 1987).

S.1 reacts with phosphorylating reagents at $\mathrm{O} 4$ and undergoes 4-triazolation in the presence of the phosphorylating reagent 2- or 4-chlorophenyl phosphorodi-1,2,4-triazolide. The triazolo derivative is converted to the fluorescent pyridinium salt $\mathbf{S . 4}$ in the presence of pyridine (Divakar and Reese, 1982; Sung, 1982; Huynh-Dinh et al., 1985). Further reaction with triazole gives $\mathbf{S . 5}$ (Fig. 2.1.3).

The purine nucleosides also react with electrophilic reagents. For example, guanine nucleosides react with MSNT to form the corresponding triazolo derivatives. Reaction of the $N^{2}$-protected guanosine nucleoside S.6 with mesitylene sulfonyl chloride gives the crystal-

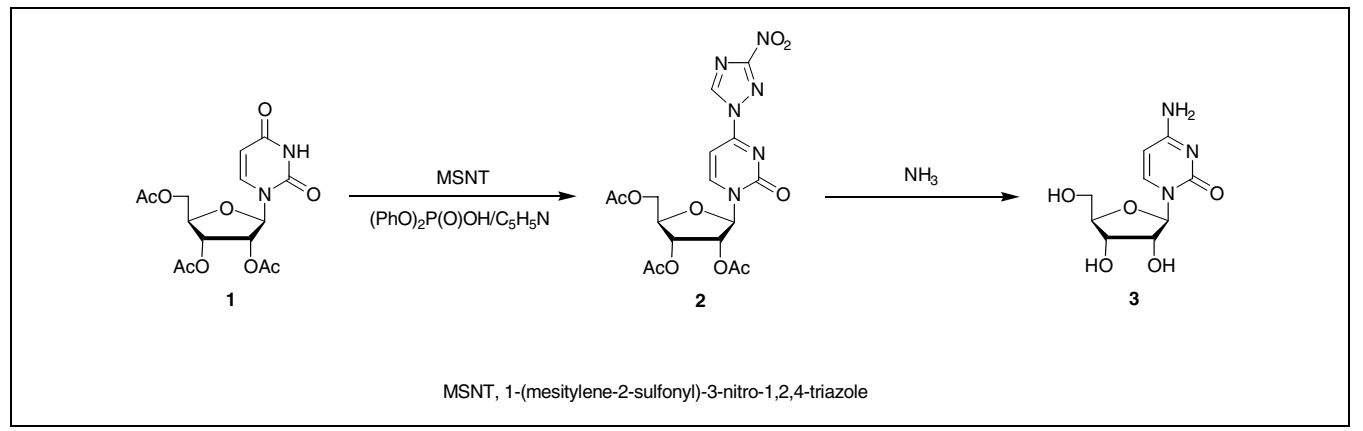

Figure 2.1.2 Conversion of tri- $O$-acetyluridine to cytidine via a nitrotriazolide intermediate. Reprinted from lyer and Beaucage (1999) with permission from Elsevier Science Publishing.

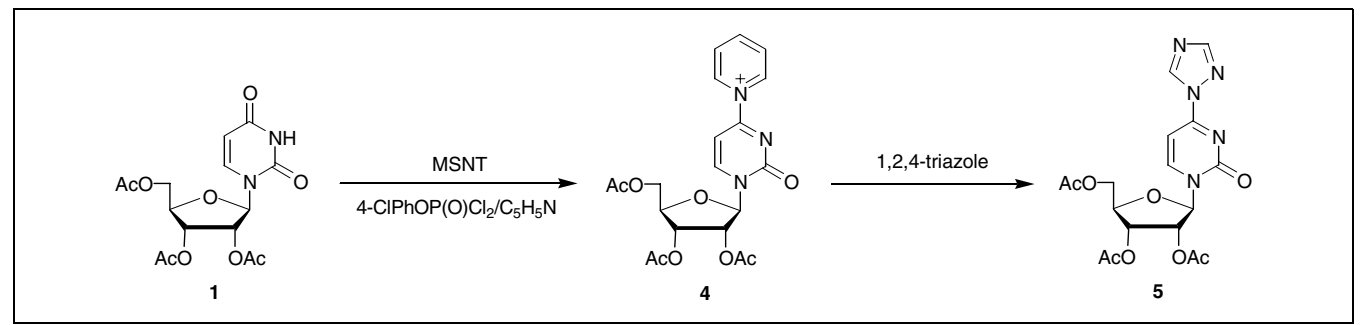

Figure 2.1.3 Formation of the triazolo derivative S.5 via the pyridinium intermediate S.4. MSNT, 1-(mesitylene-2-sulfonyl)-3-nitro-1,2,4-triazole. Reprinted from lyer and Beaucage (1999) with permission from Elsevier Science Publishing.

Protection of Nucleosides for Oligonucleotide Synthesis 


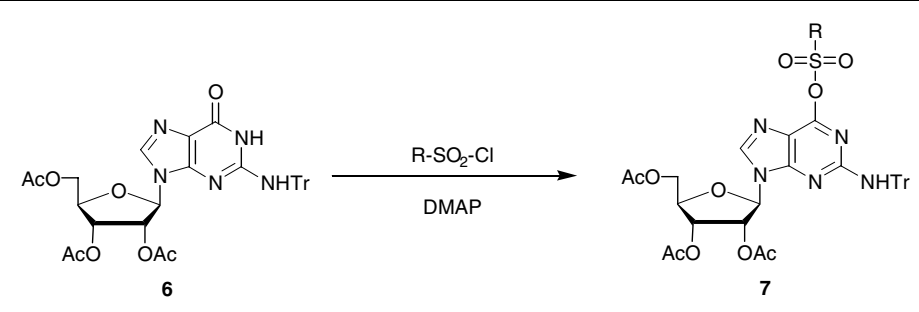

DMAP, 4-dimethylaminopyridine; Tr, triphenylmethyl

Figure 2.1.4 Formation of an $O^{6}$-sulfonated derivative of guanosine. $\mathrm{R} \cdot \mathrm{SO}_{2} \cdot \mathrm{Cl}$, mesitylene sulfonyl chloride. Reprinted from lyer and Beaucage (1999) with permission from Elsevier Science Publishing. R, 2,4,6-trimethylphenyl.

line $O^{6}$-sulfonated derivative S.7 (Fig. 2.1.4; Bridson et al., 1977; Francois et al., 1985). In the presence of pyridine, $\mathbf{S . 7}$ is converted to the corresponding C6 pyridinium compound.

The side reactions that are predominant in phosphodiester and phosphotriester chemistry have been summarized (Reese and Ubasawa, 1980). These side reactions are of concern when the $H$-phosphonate method is employed in oligonucleotide synthesis in conjunction with phosphorochloridates as coupling reagents.

The imide and lactam functions of nucleosides also react with phosphitylating reagents. For example, the 2 '-deoxyguanosine derivative S.20 (Fig. 2.1.5) reacts with methyl phosphoramidites to give the $O^{6}$-phosphitylated product S.8 (Pon et al., 1985a; Nielsen et al.,
1987). During oxidation of $\mathbf{S . 8}$ with iodine, Oto N-phosphoryl migration occurs $(\mathbf{S . 9} \rightarrow \mathbf{S . 1 0}$; Pon et al., 1985b). Once formed, S.10 induces depurination resulting in chain-cleaved products upon treatment with ammonium hydroxide during the final step of deprotection.

It is pertinent that base modifications can also occur during oligonucleotide deprotection. For example, thymine residues are significantly modified as $N^{3}$-methylthymine during deprotection of oligonucleoside methyl phosphotriesters with aqueous ammonium hydroxide (Urdea et al., 1986). However, when deprotection of the methyl phosphate backbone of oligonucleotides is accomplished by treatment with thiophenol or 2-mercaptobenzothiazole, followed by aqueous ammonium hydroxide to
Nucleobase Protection of Deoxyribo- and Ribonucleosides

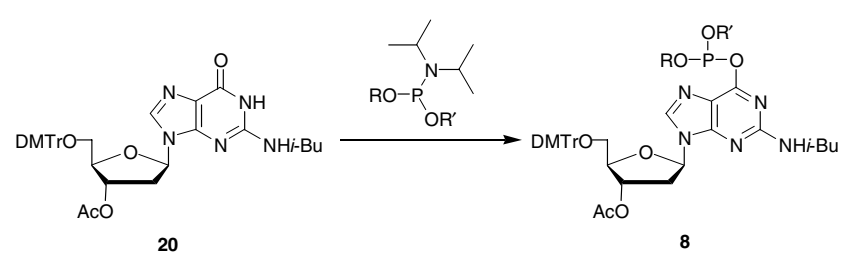

$\left[{ }^{31} \mathrm{P} \mathrm{NMR}, d 133.95,133.79 \mathrm{ppm}\right]$

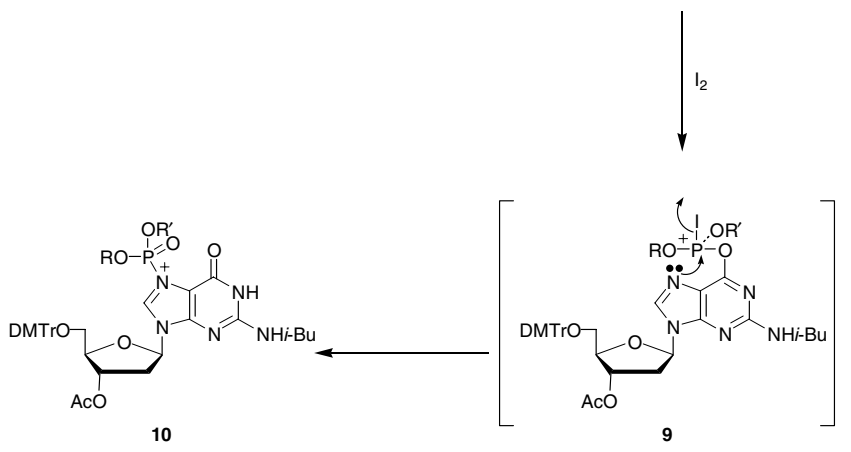

DMTr, 4,4'-dimethoxytriphenylmethyl; i-Bu, isobutyry

Figure 2.1.5 Reaction of an $N^{2}$-isobutyryl-2'-deoxyguanosine derivative with phosphitylating reagents. $R$, Me; $R^{\prime}$, nucleoside. 
remove nucleobase protecting groups, the formation of $N^{3}$-methylthymine is considerably reduced (McBride et al., 1987; Andrus and Beaucage, 1988).

The reactivity of nucleosides and the attendant risk of base modification suggest an apparent need to protect imide and lactam groups of nucleosides in oligonucleotide synthesis. It is expected that the use of protecting groups at the imide/lactam function of nucleosides can potentially avert the side reactions. This has been borne out by success in the synthesis of a number of polynucleotides (Reese, 1978).

\section{PROTECTION OF IMIDE AND LACTAM FUNCTIONS}

A number of groups have been proposed for the protection of thymine and uracil and N3 and

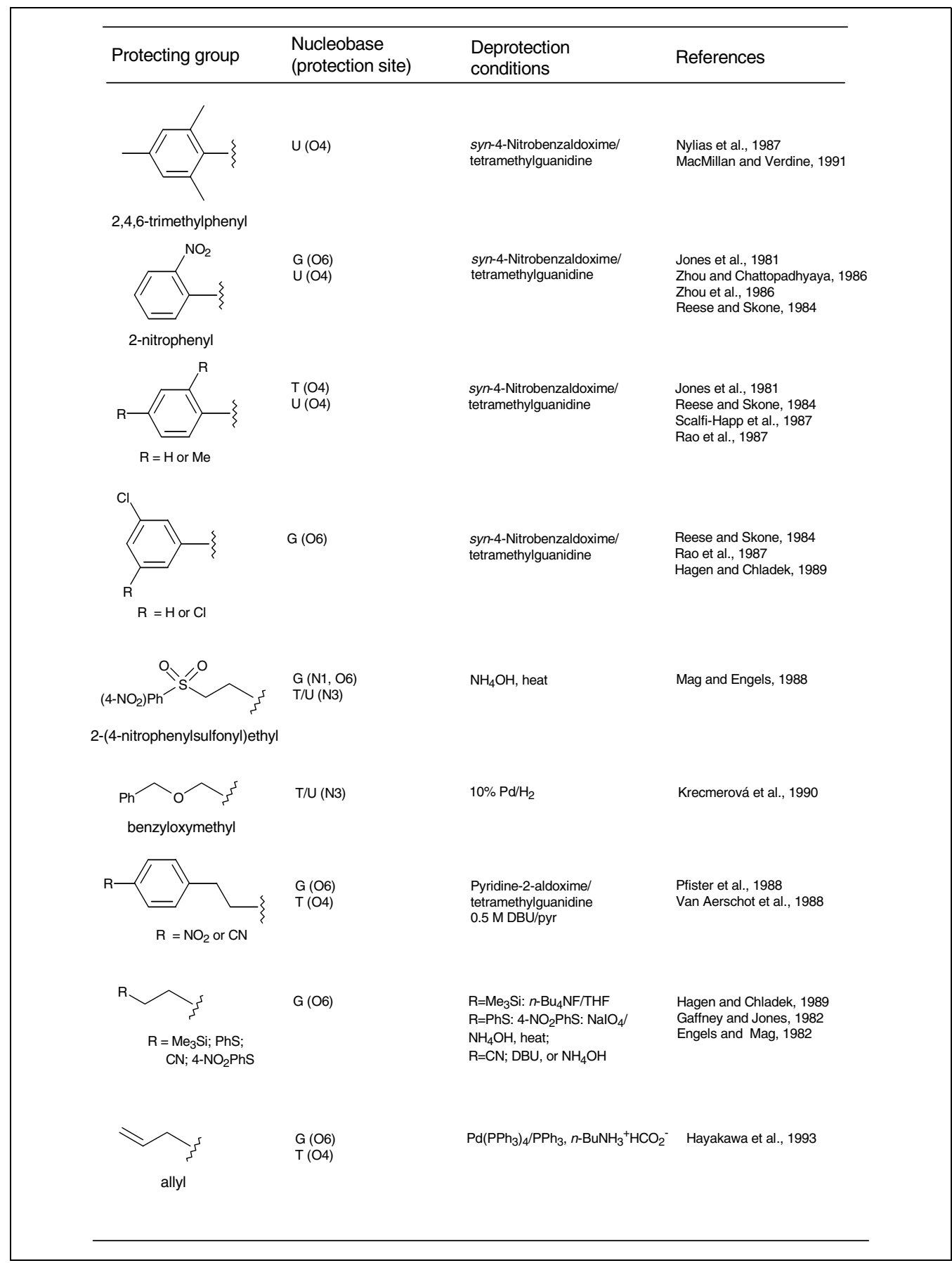

Figure 2.1.6 Protecting groups for the imide/lactam functions of guanine, uracil, and thymine. DBU, 1,8-diazabicyclo[3.4.0]undecene-7; $\mathrm{NalO}_{4}$, sodium periodate; pyr, pyridine; THF, tetrahydrofuran.
Protection of Nucleosides for Oligonucleotide Synthesis 


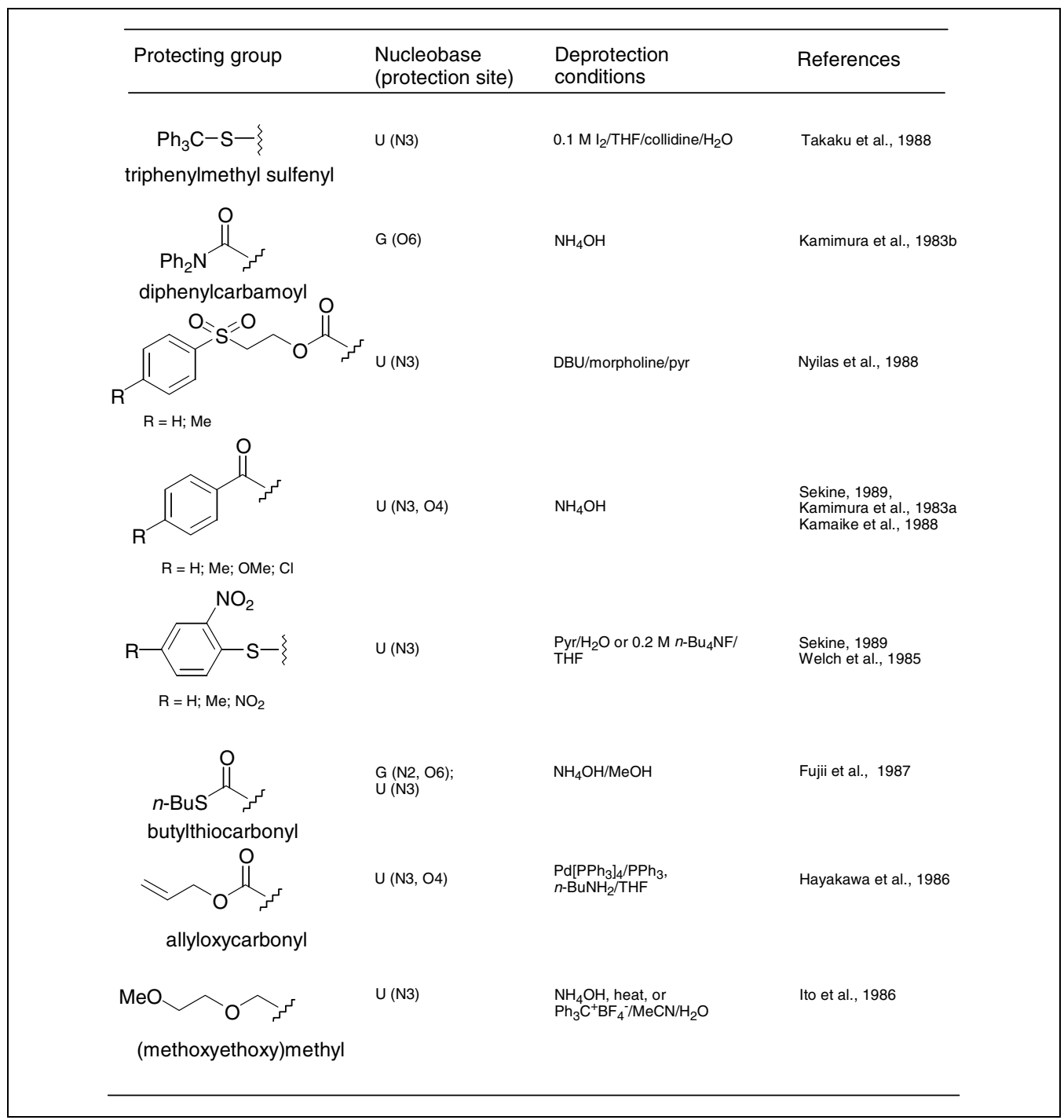

Figure 2.1.6 (Continued)

$\mathrm{O} 4$, and of guanine at $\mathrm{O} 6$. A representative list of these groups is shown in Figure 2.1.6. For the benefit of the readers, references that include experimental details are cited.

The imide- and lactam-protecting groups can be installed in two steps: sulfonylation or triazolation, followed by displacement of the resulting $O$-sulfonate or triazolide by the nucleophilic protecting group. The protecting groups can also be installed directly-for example, via Mitsunobu alkylation (UNIT 1.2) or via the transient protection approach. The preparation of these protected nucleosides appears to be straightforward (Jones et al., 1981; Gaffney and Jones, 1982; Trichtinger et al., 1983; Nyilas et al., 1987; Kamaike et al., 1988).

However, it is still a matter of debate whether imide and lactam protection is necessary in oligonucleotide synthesis. There are a number of factors to be considered. (1) As noted before, when using the phosphotriester method in oligonucleotide synthesis, $O^{6}$-modified guanine and $O^{4}$-modified uracil revert to guanine and uracil residues upon "oximate" treatment that follows chain assembly. (2) When the phosphoramidite method is used, $O^{6}$-phosphitylated deoxyguanosine reverts to deoxyguanosine on contact with water or acetate ions (Mag and Engels, 1988). Thus, during the synthesis cycle, if capping is performed (using acetic anhydride) after coupling, any $O^{6}$-modified deoxyguanosine can potentially revert back to deoxyguanosine. (3) Base modifications generated during oligonucleotide chain assembly depend on the reagents employed and the contact time. Solid-phase oligonucleotide synthesis uses automated pulsed delivery of reagents, resulting in shorter reaction times compared with solution-phase synthesis. Consequently, side 
reactions during oligonucleotide chain assembly appear to be minimal.

Thus, in principle, appropriate adjustment of synthesis protocols may obviate the necessity for imide and lactam protection. Nevertheless, $\beta$-cyanoethyl protection is generally recommended for protection of guanosine at $\mathrm{O6}$ or uridine at $\mathrm{O} 4$, and anisoyl for uridine at N3.

\section{PROTECTION OF EXOCYCLIC AMINO GROUPS}

On the basis of a number of criteria for protecting groups, as outlined previously, $\mathrm{N}$ acyl-type protection for the exocyclic amino groups has emerged as a logical choice. Figure 2.1.7 shows selected examples of $\mathrm{N}$-acyl protecting groups and the reagents used to effect their deprotection.

The $N$-acyl groups are introduced into nucleosides by a number of procedures. (1) They can be installed by peracylation of the nucleoside followed by chemoselective O-deacylation (Schaller et al., 1963). (2) The recent trend has been to use the "transient" protection approach (Ti et al., 1982). In this procedure, the nucleoside is persilylated using a silylating agent, and the acyl function is installed on the amino group using the corresponding acid chloride or acid anhydride. (3) Some acyl functions such as benzoyl, $\alpha$-phenylcinnamoyl, and naphthaloyl are directly incorporated on the nucleobase using the corresponding anhydride (Watanabe and Fox, 1966; Bhat et al., 1989). (4) The exocyclic amino group of cytidine and deoxycytidine can be directly acylated using activated esters (Igolen and Morin, 1980), acid chlorides (Mishra and Misra, 1986, and references therein), or alkyloxycarbonylbenzotriazoles (Himmelsbach et al., 1984). (5) Recent reports suggest that site-selective incorporation

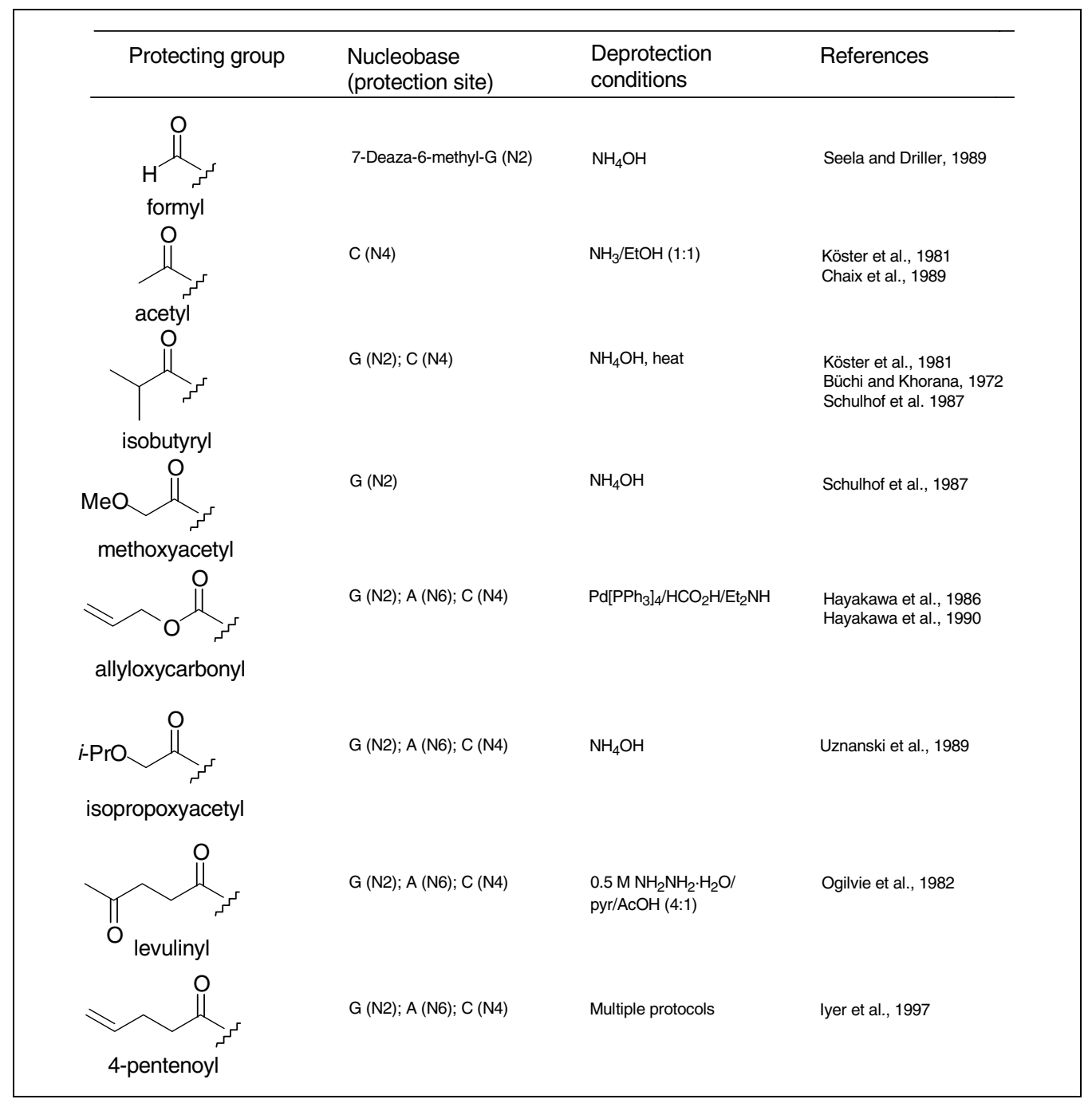

Figure 2.1.7 Protecting groups for the exocyclic amino function of nucleobases. RT, room temperature; pyr, pyridine.
Protection of Nucleosides for Oligonucleotide Synthesis 


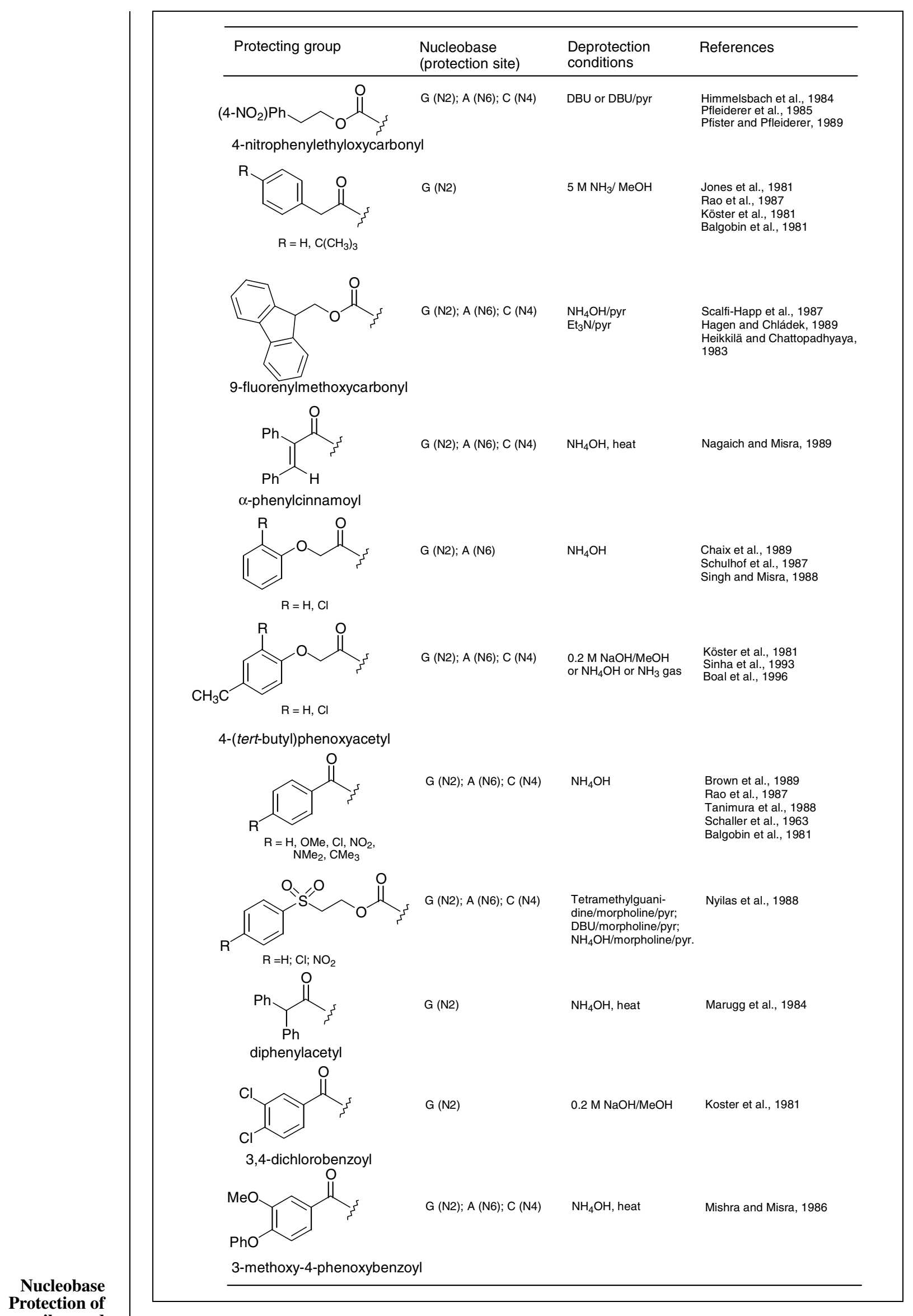

Protection of

Deoxyribo- and Ribonucleosides

Figure 2.1.7 (Continued) 


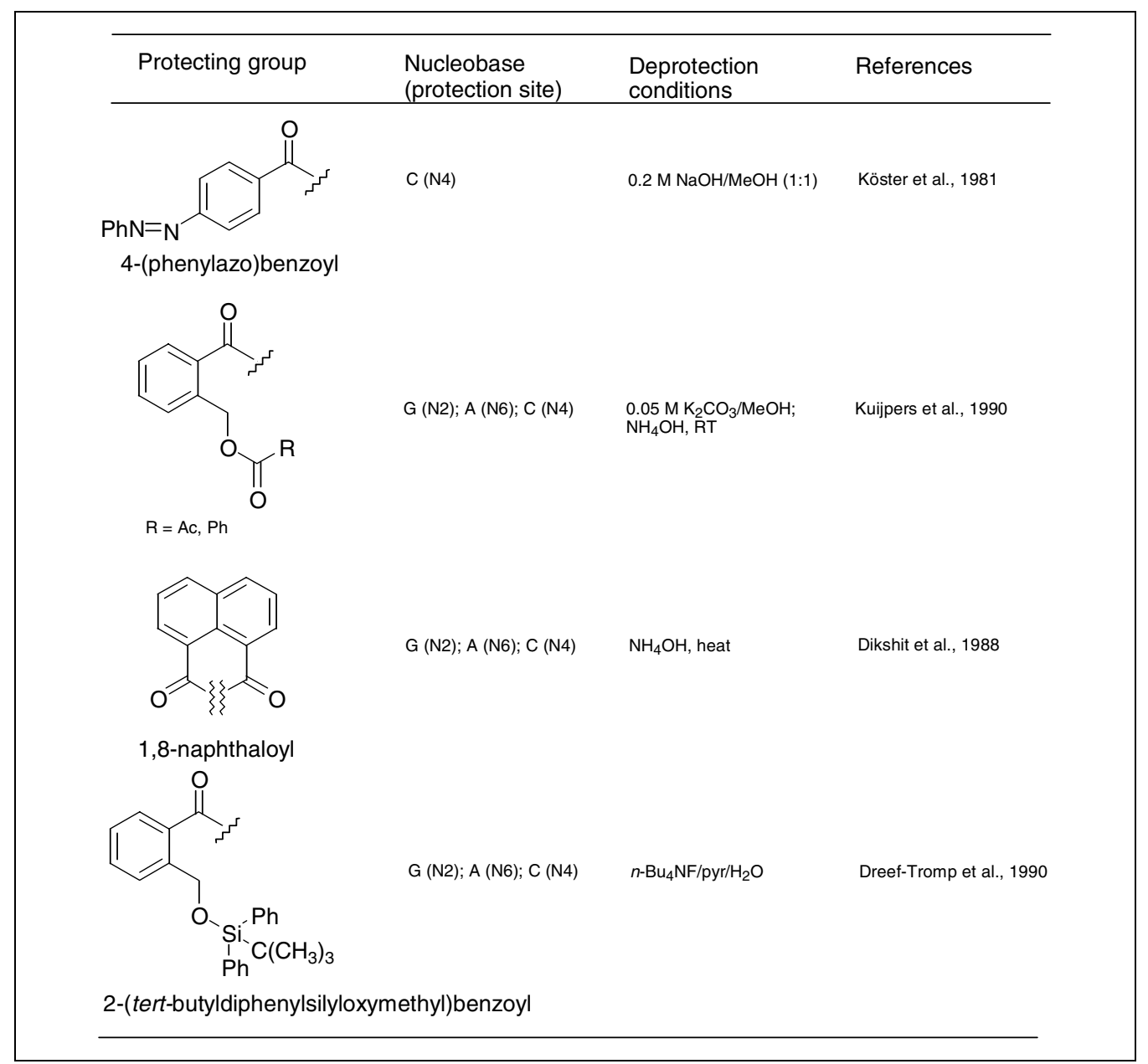

Figure 2.1.7 (Continued)

and removal of $\mathrm{N}$-acyl protecting groups can also be achieved by enzymatic methods (Prasad and Wengel, 1996).

Overall, most $N$-acyl protecting groups are stable in neutral or acidic medium and moderately stable at high $\mathrm{pH}(\mathrm{pH}>13)$. However, many $N$-acyl protecting groups are readily removed by ammonolysis. This observation forms the basis for the widespread use of $28 \%$ $\mathrm{NH}_{4} \mathrm{OH}$ as a deprotection reagent when $N$-acylprotected nucleosides are employed in oligonucleotide synthesis.

A salient feature of $\mathrm{N}$-acyl protecting groups is that their stability in alkaline $\mathrm{pH}$ can be modulated by the steric and electronic characteristics of specific acyl groups. A study comparing the stability of various $\mathrm{N}$-acyl nucleosides in alkaline medium $(0.2 \mathrm{~N} \mathrm{NaOH} / \mathrm{MeOH})$ has been reported (Köster et al., 1981). Importantly, the stability of the acyl function towards alkaline hydrolysis is determined by the nature of the heterocyclic base. For example, the rate of deacylation of $N$-acyl derivatives of deoxycytidine is faster than that of deoxyadenosine or deoxyguanosine. The hydrolytic lability is also determined by inductive, resonance, and steric effects. For example, in a series of $N$-acyl nucleosides, $N$-benzoyl nucleosides are hydrolyzed sixteen times faster than $N$-(2,4-dimethyl)benzoyl nucleosides, presumably because of steric effects. Similarly, $N$ - $(2,4-$ dimethoxy)benzoyl nucleoside is hydrolyzed eight times faster than $N$-(4-dimethylamino)benzoyl nucleoside, perhaps due to a combination of inductive and resonance effects.

The choice of a particular $N$-acyl protecting group also depends on the type of coupling chemistry that is employed. For example, when phosphodiester and phosphotriester chemistries are used in oligonucleotide synthesis, it is necessary to select sturdy $\mathrm{N}$-acyl protecting groups that can withstand the harsh reagents and conditions employed during synthesis. This requirement is met by the benzoyl group for adenine and cytosine, and the isobutyryl group for guanine. However, removal of these protecting groups requires prolonged heating
Protection of Nucleosides for Oligonucleotide Synthesis 
(12 to $14 \mathrm{hr}$ ) with $28 \% \mathrm{NH}_{4} \mathrm{OH}$ at $55^{\circ} \mathrm{C}$. In spite of this limitation, these protecting groups have remained popular even with the advent of automated solid-phase oligonucleotide synthesis using phosphoramidite and $H$-phosphonate chemistries.

\section{PROTECTION OF PURINE NUCLEOBASES: THE PROBLEM OF DEPURINATION}

The development of suitable protecting groups for purine nucleobases has been an area of considerable interest because purine nucleosides rapidly depurinate under acidic conditions. The problem is compounded by the acidlabile DMTr group used for protection of the 5 '-OH in solid-phase oligonucleotide synthesis. Prior to each coupling step in the synthesis cycle, the DMTr group is removed by exposure to a strong acid such as $2 \%$ dichloroacetic acid in dichloromethane. Consequently, the growing oligonucleotide chain is repeatedly exposed to strongly acidic conditions, potentially resulting in depurination and reduced yield of the desired "full-length" product.

The kinetics and mechanisms of nucleoside depurination have been investigated by several research groups (Romero et al., 1978; Oivanen et al., 1987; Suzuki et al., 1994). The presumed mechanism for depurination of deoxyadenosine (Fig. 2.1.8) involves initial protonation of the nucleoside to produce the $N^{1}$-protonated form S.11, and then equilibration (prototropic shift) to the $N^{7}$-protonated species $\mathbf{S . 1 2}$ or $\mathbf{S . 1 3}$, followed by cleavage of the glycosidic bond to give S.15 via the oxonium S.14 (Zoltewicz et al., 1970; Zoltewicz and Clark, 1972). It is also conceivable that, at lower $\mathrm{pH}$, depurination can occur via protonation of the purine nucleobase at both $\mathrm{N} 1$ and N7. Presence of the $2^{\prime}-\mathrm{OH}$ has a significant effect on the nucleoside's susceptibility to depurination. For example, guanosine and adenosine are more resistant to depurination compared with deoxyadenosine and deoxyguanosine. Deoxyadenosine itself depurinates 1200 times faster than adenosine (York, 1981).

Interestingly, $\mathrm{N}$-acyl-protected purine nucleosides (particularly deoxyadenosine) are more prone to depurination than unprotected nucleosides. Among $N$-acyl-protected deoxyadenosines, protection at N6 with $\alpha$ phenylcinnamoyl, naphthaloyl, 3-methoxy-4phenoxybenzoyl, 9-fluorenylmethoxycarbonyl (FMOC), and tert-butylphenoxyacetyl $(t$-PAC) groups (Fig. 2.1.7) provides greater resistance to depurination than with $N^{6}$-benzoyl (reviewed in Beaucage and Iyer, 1992). It is believed that in the case of acyl-protected
Nucleobase Protection of Deoxyribo- and Ribonucleosides

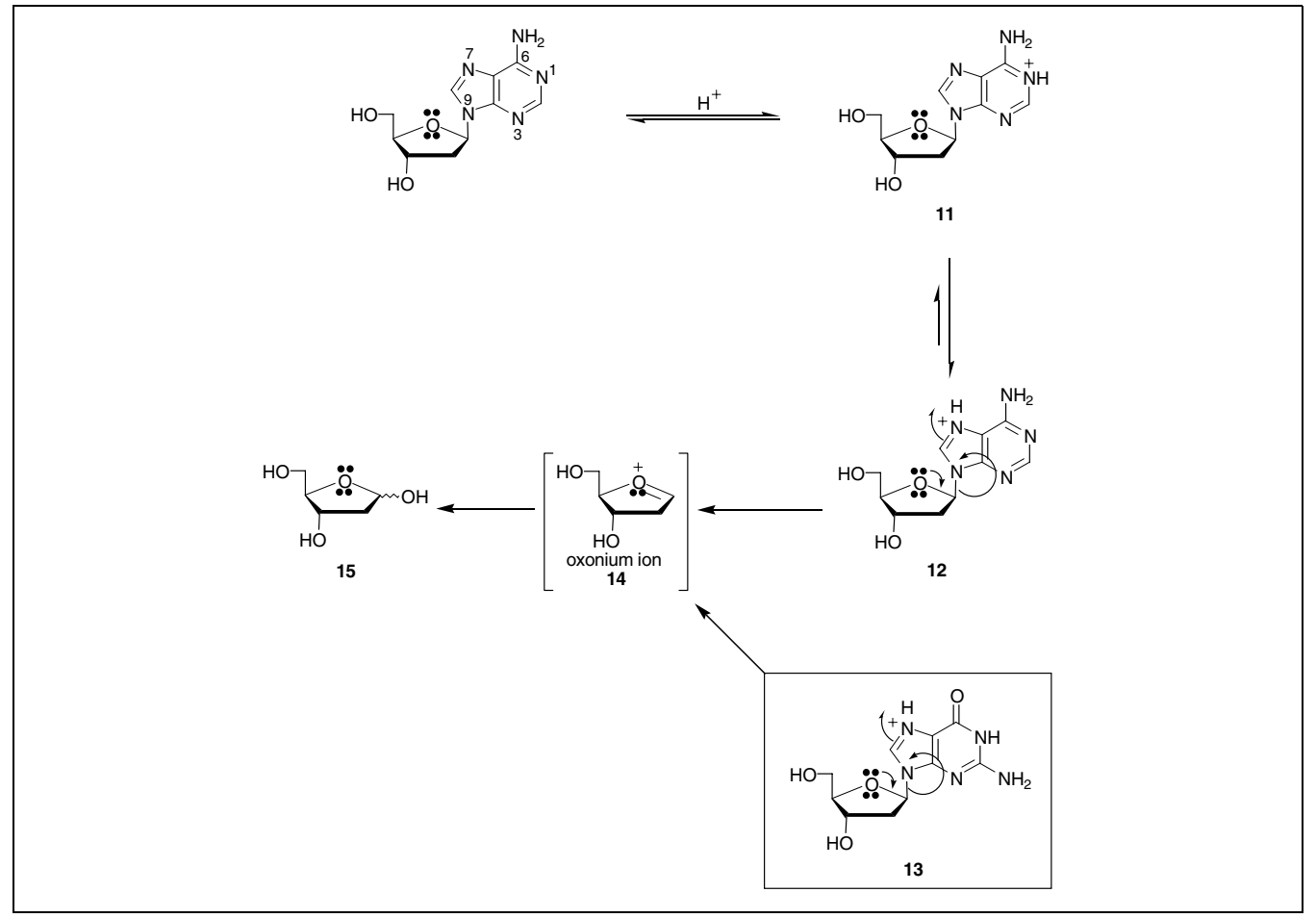

Figure 2.1.8 Scheme showing the proposed depurination mechanism for 2 '-deoxyguanosine and 2'-deoxyadenosine catalyzed by protic acids. Modified from lyer and Beaucage (1999) with permission from Elsevier Science Publishing. 

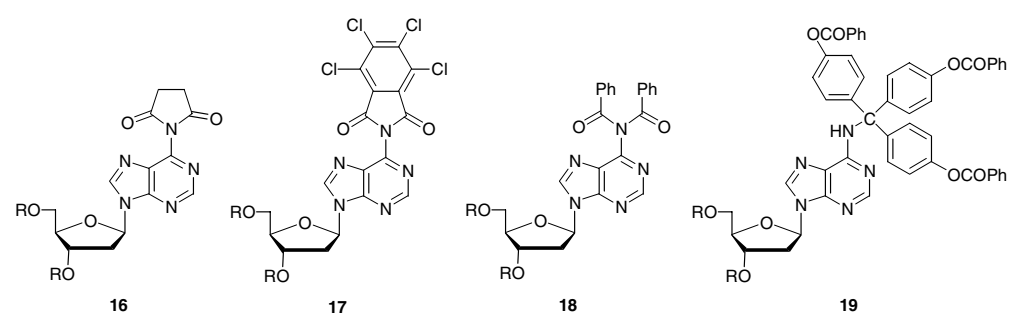

Figure 2.1.9 Examples of $N^{6}$-protecting groups for $2^{\prime}$-deoxyadenosine derivatives that reduce depurination. Reprinted from lyer and Beaucage (1999) with permission from Elsevier Science Publishing. R, DMTr.

purine nucleosides under acidic conditions, the initial site of protonation is $\mathrm{N} 7$, rendering the protonated species more prone to glycosidic cleavage. Naturally, caution should be exercised in the synthesis of oligonucleotides whose sequence contains deoxyadenosines at the 3 ' terminus.

Bis-acylation has also been studied as a strategy to reduce depurination. Imide protecting groups $\mathbf{S . 1 6}$ and $\mathbf{S . 1 7}$, as well as the diamide protecting group $\mathbf{S . 1 8}$, have been investigated (Fig. 2.1.9; Kume et al., 1982, 1984). However, these groups were labile to aqueous pyridine, a solvent used during the oxidation step in solidphase oligonucleotide synthesis. Thus, alternate oxidants have to be employed for the oxidation step.

Since $N$-acyl-protected purine nucleosides are sensitive to deacylation, alternate protecting groups have been investigated. Prominent among these are the amidine protecting groups, which are introduced using an exchange reaction with appropriate amidine acetals. Interestingly, $\mathrm{N}$-amidine-protected nucleosides (Fig. 2.1.10) resist depurination 20 -fold better than the corresponding $\mathrm{N}$-benzoyl nucleosides

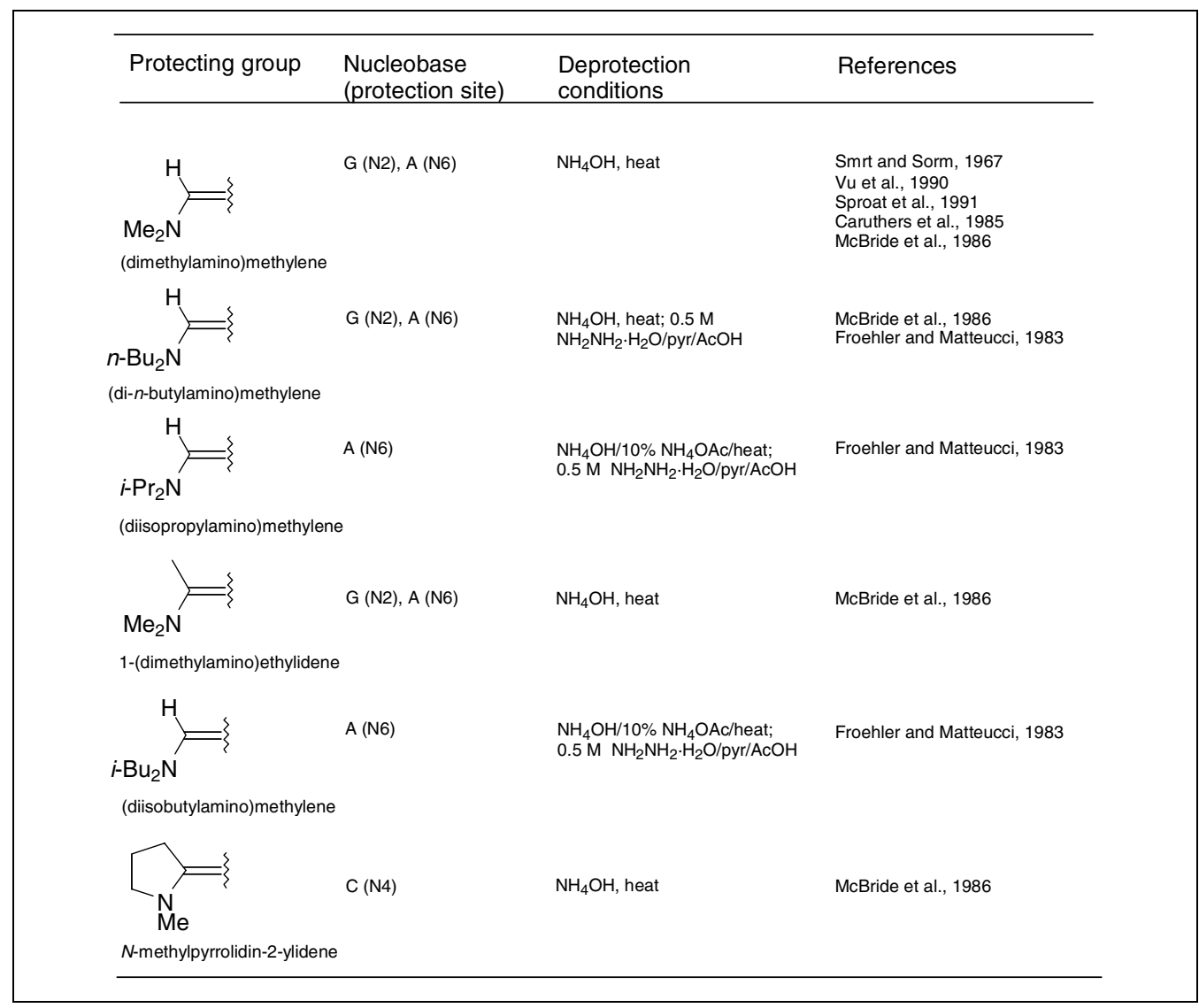

Figure 2.1.10 Amidine protecting groups for exocyclic amino functions. Pyr, pyridine.
Protection of Nucleosides for Oligonucleotide Synthesis 
(Smrt and Sorm, 1967; Holy and Zemlicka, 1969, and references therein; Froehler and Matteucci, 1983; Caruthers et al., 1985; Vu et al., 1990; Sproat et al., 1991).

It is presumed that the protonation sites of amidine-protected nucleosides are $\mathrm{N} 1$ and $\mathrm{N} 6$ instead of $\mathrm{N} 1$ and $\mathrm{N} 7$, respectively, resulting in a slower rate of depurination. Indeed, amidineprotected nucleoside phosphoramidites are frequently employed in solid-phase oligonucleotide synthesis. However, changes are required in the oxidation step to avoid nucleobase modifications (Mullah et al., 1995).

Nucleosides protected with $O$-nitrophenylsulfonyl and tris(benzoyloxy)trityl (S.19) groups also appear to be more resistant to depurination (Shimidzu and Letsinger, 1968; Honda et al., 1984, and references therein). However, the derived nucleoside phosphoramidites couple less efficiently than the $N$-acyl-protected nucleoside phosphoramidites (Sekine et al., 1985).

Depurination is also influenced by other factors such as the nature of the solid support (controlled-pore glass versus polystyrene; see UNIT 3.1), the composition of the deblocking solution and deblocking time, and the washing solvent and washing time that are employed in solid-phase synthesis of oligonucleotides (Paul and Royappa, 1996). Depurination is faster at terminal sites than at internal sites in an oligonucleotide chain (Suzuki et al., 1994). Interestingly, a solution of $15 \%$ dichloroacetic acid (DCA) in methylene chloride was ideal as a detritylating reagent that induced minimal depurination compared to the traditionally used $2 \% \mathrm{DCA} /$ methylene chloride.

It is pertinent that with modern DNA synthesizers the pulsed delivery of reagents to the synthesis columns, in conjunction with optimized synthesis programs, results in short contact times and has greatly minimized the depurination problem. Thus, $\mathrm{N}$-acyl-protected nucleoside (benzoyl for $\mathrm{dA}$ and $\mathrm{dC}$, and isobutyryl for $\mathrm{dG}$ ) phosphoramidites and $H$-phosphonates can be used for the efficient synthesis of oligonucleotides.

\section{RECENT TRENDS IN NUCLEOBASE PROTECTION}

Over the past few years, new applications of oligonucleotides in diagnostics and therapeutics have emerged, necessitating the expeditious synthesis of large numbers of oligonucleotides. In order to speed the synthesis process, more "labile" $N$-acyl protecting groups for nucleosides have been sought. As the simplest member of the family of $N$-acyl protecting groups, the $N^{4}$-acetyl of cytosine has been used for "ultrafast" DNA synthesis using the phosphoramidite approach. Rapid deprotection rates were achieved using methylamine/ammonia (Reddy et al., 1994). This group is unsuitable for use in phosphodiester and phosphotriester chemistries, however.

The synthesis of oligonucleotide analogs carrying sensitive backbones requires protecting groups that (1) withstand the synthetic rigors of chain assembly, and (2) can be removed chemoselectively under mild conditions. Nucleobases protected by phenoxyacetyl (Singh and Misra, 1988; Chaix et al., 1989; Sproat et al., 1991), and their derivatives such as $t$-PAC, show accelerated deacylation (Köster et al., 1981; Sinha et al., 1993) under mildly basic conditions. It is presumed that the inductive effect of the phenoxy group renders the amide carbonyl group more susceptible to nucleophilic attack, facilitating rapid base-catalyzed hydrolysis. Thus, PAC- and $t$-PAC-phosphoramidites have been employed for rapid synthesis of oligonucleotides and certain analogs. As a rule, following oligonucleotide assembly on solid support, the PAC and $t$-PAC groups are removed under milder conditions (28\% $\mathrm{NH}_{4} \mathrm{OH}$, room temperature; Sinha et al., 1993) or, according to a recent report, using gaseous amines under pressure (Boal et al., 1996). However, the use of $t$-PAC-protected nucleoside phosphoramidites results in trans acylation of the $t$-PAC protecting groups during the capping step when acetic anhydride is employed as a capping reagent. Thus, tert-butylphenoxyacetic anhydride should be used for the capping step in solid-phase oligonucleotide synthesis (Sinha et al., 1993).

The concept of neighboring-group participation has also been used to design acyl protecting groups and to accelerate the deprotection step (Dreef-Tromp et al., 1990; Kuijpers et al., 1990). For example, removal of the 2-(acetoxymethyl)benzoyl group from nucleosides under basic conditions is accelerated by intramolecular participation of the deacylated hydroxymethyl group.

New protecting groups have also been introduced that can be chemoselectively removed under neutral or mildly basic conditions. Indeed, the allyloxycarbonyl protecting group (Hayakawa et al., 1986, 1990) is chemoselectively removed using $\operatorname{Pd}(0)$, whereas the ( $p$-nitrophenyl)ethoxycarbonyl group (Trichtinger et al., 1983; Pfleiderer et al., 1985; Pfister et al., 1988) and the 2-dansylethoxy cabonyl group 
(Wagner and Pfleiderer, 1997) are selectively removed using DBU. Consequently, supportbound oligonucleotides can be prepared using building blocks that carry these protecting groups. Recently, the $N$-pent-4-enoyl (PNT) group has been introduced as a new acyl protecting group for situations where multiple deprotection protocols are used (Iyer et al., 1997; see also references therein). PNT-protected nucleoside phosphoramidites can potentially be used for the rapid synthesis of oligonucleotides and oligonucleotide analogs, as well as for the preparation of support-bound oligonucleotides.

Several groups have been reported for nucleobase protection and may be particularly valuable in the synthesis of oligonucleotides intended for specific applications. Figure 2.1.7 shows a partial list of protecting groups. A complete list of such groups is covered in other reviews (Sonveaux, 1986; Beaucage and Iyer, 1992; Iyer and Beaucage, 1999). Nonetheless, it should be noted that many of these still need to be evaluated in routine synthesis.

Because of various issues associated with nucleobase protection, oligonucleotide synthesis has been evaluated using building blocks bearing unprotected nucleobases. However, these efforts have met with only limited success (Narang et al., 1972; Fourrey and Varenne, 1985; Gryaznov and Letsinger, 1991; Uchiyama et al., 1993, and references therein). Clearly, more work is necessary in this area.

\section{CONCLUSION}

Evidently, a nucleobase protecting group should meet several criteria before it can be adapted for routine oligonucleotide synthesis. It is imperative, therefore, that the potential for side reactions be closely examined when designing new reagents, evaluating new protecting groups, and implementing modifications of established protocols during oligonucleotide synthesis, as well as during the manufacture of oligonucleotides and their analogs.

The development of nucleobase protecting groups and deprotection protocols has been crucial for the successful synthesis of oligonucleotides, functionalized oligonucleotides, oligonucleotide analogs, ribonucleotides, and phosphorylated biomolecules (reviewed in Beaucage and Iyer, 1992, 1993a,b,c). As in the past, oligonucleotides are expected to play a dominant role in fostering advances in functional genomics, proteomics, diagnostics, and therapeutics. Consequently, continued demand exists for simultaneous synthesis of large numbers of oligonucleotides in miniature formats, and for the synthesis and manufacture of novel analogs. It is hoped that the present commentary will serve as a framework for developing new protecting group strategies for oligonucleotide synthesis that could meet these challenges.

\section{ACKNOWLEDGMENT}

The author wishes to thank Dr. WenQiang Zhou for his help with the artwork in this manuscript.

\section{LITERATURE CITED}

Andrus, A. and Beaucage, S.L. 1988. 2-Mercaptobenzothiazole - an improved reagent for the removal of methylphosphate protecting groups from oligodeoxynucleotide phosphotriesters. Tetrahedron Lett. 29:5479-5482.

Balgobin, N., Josephson, S., and Chattopadhyaya, J.B. 1981. A general approach to the chemical synthesis of oligodeoxyribonucleotides. Acta. Chem. Scand. B35:201-212.

Beaucage, S.L. and Caruthers, M.H. 1981. Deoxynucleoside phosphoramidites-A new class of key intermediates for deoxypolynucleotide synthesis. Tetrahedron Lett. 22:1859-1862.

Beaucage, S.L. and Iyer, R.P. 1992. Advances in the synthesis of oligonucleotides by the phosphoramidite approach. Tetrahedron 48:22232311.

Beaucage, S.L. and Iyer, R.P. 1993a. The functionalization of oligonucleotides via phosphoramidite derivatives. Tetrahedron 49:19251963.

Beaucage, S.L. and Iyer, R.P. 1993b. The synthesis of modified oligonucleotides by the phosphoramidite approach and their applications. Tetrahedron 49:6123-6194.

Beaucage, S.L. and Iyer, R.P. 1993c. The synthesis of specific ribonucleotides and unrelated phosphorylated biomolecules by the phosphoramidite method. Tetrahedron 49:10441-10488.

Bhat, V., Ugarkar, B.G., Sayeed, V.A., Grimm, K., Kosora, N., and Domenico, P. 1989. A simple and convenient method for the selective $\mathrm{N}$-acylations of cytosine nucleosides. Nucleosides $\mathrm{Nu}$ cleotides 8:179-183.

Boal, J.H., Wilk, A., Harindranath, N., Max, E.E., Kempe, T., and Beaucage, S.L. 1996. Cleavage of oligodeoxyribonucleotides from controlledpore glass supports and their rapid deprotection by gaseous amines. Nucl. Acids Res. 24:31153117.

Bridson, P.K., Markiewicz, W., and Reese, C.B. 1977. Acylation of 2',3',5'-tri-O-acetylguanosine. J. Chem. Soc., Chem. Commun. 791-792.

Brown, J.M., Christodoulou, C., Modak, A.S., Reese, C.B., and Serafinowska, H.T. 1989. Synthesis of the $3^{\prime}$-terminal half of yeast alanine transfer ribonucleic acid (tRNA ${ }^{\text {ala }}$ ) by the phosphotriester approach in solution. Part 2. J. Chem. Soc. Perkin Trans. 1:1751-1767.
Protection of Nucleosides for Oligonucleotide Synthesis 
Büchi, H. and Khorana, H.G. 1972. CV. Total synthesis of the structural gene for an alanine transfer ribonucleic acid from yeast. Chemical synthesis of an icosadeoxyribonucleotide corresponding to the nucleotide sequence 31 to $50 . J$. Mol. Biol. 72:251-288.

Caruthers, M.H., McBride, L.J., Bracco, L.P., and Dubendorff, J.W. 1985. Studies on nucleotide chemistry 15 . Synthesis of oligodeoxynucleotides using amidine protected nucleosides. Nucleosides Nucleotides 4:95-105.

Chaix, C., Molko, D., and Téoule, R. 1989. The use of labile base protecting groups in oligoribonucleotide synthesis. Tetrahedron Lett. 30:71-74.

Clauwaert, J. and Stockx, J. 1986. Interactions of polynucleotides and their components. I. Dissociation constants of the bases and their derivatives. Z. Naturforsch. B. 23:25-30.

den Hartog, J.A.J., Willie, G., Scheublin, R.A., and van Boom, J.H. 1982. Chemical synthesis of a messenger ribonucleic acid fragment: AUGUUCUUCUUCUUCUUC. Biochemistry 21:10091018.

Dikshit, A., Chaddha, M., Singh, R.K., and Misra, K. 1988. Naphthaloyl group: A new selective amino protecting group for deoxynucleosides in oligonucleotide synthesis. Can. J. Chem. 66:2989-2994.

Divakar, K.J. and Reese, C.B. 1982. 4-(1,2,4-Triazol-1-yl)- and 4-(3-nitro-1,2,4-triazol-1-yl)-1( $\beta$-D-2,3,5-tri-O-acetylarabinofuranosyl)pyrim idin-2(1H)-ones. Valuable intermediates in the synthesis of derivatives of 1-( $\beta$-D-arabinofuranosyl)cytosine (Ara-C). J. Chem. Soc. Perkin Trans. 1:1171-1176.

Dreef-Tromp, C.M., van Dam, E.M.A., van den Elst, H., van der Marel, G.A., and van Boom, J.H. 1990. Solid-phase synthesis of H-Phe-Tyr(pATAT)- $\mathrm{NH}_{2}$ : A nucleopeptide fragment from the nucleoprotein of bacteriophage $\phi X 174$. Nucl. Acids Res. 18:6491-6495.

Dunn, D.B., and Hall, R.H. 1975. Purines, pyrimidines, nucleosides and nucleotides: Physical constants and spectral properties. In Handbook of Biochemistry and Molecular Biology, 3rd ed., Vol. 1: Nucleic Acids (G.D. Fasman, ed.) pp. 65-125. CRC Press, Boca Raton, Fla.

Engels, J.W. and Mag, M. 1982. Amide protection in oligodeoxynucleotide synthesis. Nucleosides Nucleotides 6:473-475.

Fourrey, J.-L. and Varenne, J. 1985. Preparation and phosphorylation reactivity of $N$-nonacylated nucleoside phosphoramidites. Tetrahedron Lett. 26:2663-2666.

Francois, P., Hamoir, G., Sonveaux, E., Vermeersch, H., and Ma, Y. 1985. On the phosphorylation of deoxyribonucleosides and the protection of deoxyguanosine. Bull. Soc. Chim. Belg. 94:821823.

Nucleobase Protection of Deoxyribo- and Ribonucleosides
Froehler, B.C. and Matteucci, M.D. 1983. Dialkylformamidines: Depurination resistant $\mathrm{N}^{6}$-protecting group for deoxyadenosine. Nucl. Acids Res. 11:8031-8036.
Froehler, B.C., Ng, P.G., and Matteucci, M.D. 1986. Synthesis of DNA via deoxynucleoside H-phosphonate intermediates. Nucl. Acids Res. 14:5399-5407.

Fujii, M., Yamakage, S., Takaku, H., and Hata, T. 1987. (Butylthio)carbonyl group: A new protecting group for the guanine residue in oligoribonucleotide synthesis. Tetrahedron Lett. 28:57135716.

Gaffney, B.L. and Jones, R.A. 1982. A new strategy for the protection of deoxyguanosine during oligonucleotide synthesis. Tetrahedron Lett. 23:2257-2260.

Garegg, P.J., Lindh, I., Regberg, T., Stawinski, J., Strömberg, R., and Henrichson, C. 1986. Nucleoside H-phosphonates. IV. Automated solid phase synthesis of oligoribonucleotides by the hydrogenphosphonate approach. Tetrahedron Lett. 27:4055-4058.

Gilham, P.T. and Khorana, H.G. 1958. Studies on polynucleotides. I. A new and general method for the chemical synthesis of the $\mathrm{C}_{5}{ }^{\prime}-\mathrm{C}_{3}{ }^{\prime}$ internucleotidic linkage. Syntheses of deoxyribo-dinucleotides. J. Am. Chem. Soc. 80:6212-6222.

Gryaznov, S.M. and Letsinger, R.L. 1991. Synthesis of oligonucleotides via monomers with unprotected bases. J. Am. Chem. Soc. 113:5876-5877.

Hagen, M.D. and Chládek, S. 1989. General synthesis of $2^{\prime}\left(3^{\prime}\right)-O$-aminoacyl oligoribonucleotides. The protecion of the guanine moiety. J. Org. Chem. 54:3189-3195.

Hall, R.H., Todd, A.R., and Webb, R.F. 1957. Nucleotides. Part XLI. Mixed anhydrides as intermediates in the synthesis of dinucleoside phosphates. J. Chem. Soc. 3291-3296.

Hayakawa, Y., Kato, H., Uchiyama, M., Kajino, H., and Noyori, R. 1986. Allyloxycarbonyl group: A versatile blocking group for nucleotide synthesis. J. Org. Chem. 51:2400-2402.

Hayakawa, Y., Wakabayashi, S., Kato, H., and Noyori, R. 1990. The allylic protection method in solid-phase oligonucleotide synthesis. An efficient preparation of solid-anchored DNA oligomers. J. Am. Chem. Soc. 112:1691-1696.

Hayakawa, Y., Hirose, M., and Noyori, R. 1993. $O$-Allyl protection of guanine and thymine residues in oligodeoxyribonucleotides. J. Org. Chem. 58:5551-5555.

Heikkilä, J. and Chattopadhyaya, J. 1983. The 9fluorenylmethoxycarbonyl (Fmoc) group for the protection of amino functions of cytidine, adenosine, guanosine and their $2^{\prime}$-deoxysugar derivatives. Acta Chem. Scand. B37:263-265.

Himmelsbach, F., Schulz, B.S., Trichtinger, T., Charubala, R., and Pfleiderer, W. 1984. The $p$ nitrophenylethyl (NPE) group. A versatile new blocking group for phosphate and aglycone protection in nucleosides and nucleotides. Tetrahedron 40:59-72. 
Holy, A. and Zemlicka, J. 1969. Oligonucleotidic compounds. XXXIII. A study on hydrolysis of $\mathrm{N}$-dimethylaminomethylenecytidine, -adenosine, -guanosine, and related 2'-deoxy compounds. Collect. Czech. Chem. Commun. 34:2449-2458.

Honda, S., Urakami, K., Koura, K., Terada, K., Sato, Y., Kohno, K., Sekine, M., and Hata, T. 1984. Synthesis of oligoribonucleotides by use of $S, S$ diphenyl $N$-monomethoxytrityl ribonucleoside 3'-phosphorodithioates. Tetrahedron 40:153163.

Huynh-Dinh, T., Langlois d'Estaintot, B., Allard, P., and Igolen, J. 1985. Synthèse simplifiée de sondes mixtes avec des triazolo-nucléosides. Tetrahedron Lett. 26:431-434.

Igolen, J. and Morin, C. 1980. Rapid synthesis of protected 2'-deoxycytidine derivatives. J. Org. Chem. 45:4802-4804.

Ito, T., Ueda, S., and Takaku, H. 1986. (Methoxyethoxy)methyl group: New amide and hydroxyl protecting groups of uridine in oligonucleotide synthesis. J. Org. Chem. 51:931933.

Iyer, R.P. and Beaucage, S.L. 1999. Oligonucleotide synthesis. In Comprehensive Natural Products Chemistry, Vol. 7: DNA and Aspects of Molecular Biology (E.T. Kool, ed.) pp. 105-152. Elsevier Science Publishing, New York.

Iyer, R.P., Yu, D., Habus, I., Ho, N.H., Johnson, S., Devlin, T., Jiang, Z., Zhou, W., Xie, J., and Agrawal, S. 1997. N-Pent-4-enoyl (PNT) group as a universal nucleobase protector: Applications in the rapid and facile synthesis of oligonucleotides, analogs and conjugates. Tetrahedron 53:2731-2750.

Jones, S.S., Reese, C.B., Sibanda, S., and Ubasawa, A. 1981. The protection of uracil and guanine residues in oligonucleotide synthesis. Tetrahedron Lett. 22:4755-4758.

Kamaike, K., Hasegawa, Y., and Ishido, Y. 1988. A simple, preparative procedure for $N^{3}$-anisoyluridine and $O^{6}$-diphenylcarbamoylguanosine $2^{\prime}$ $O$-(tetrahydropyran-2-yl) derivatives via the corresponding 3',5'-dibenzoates. Nucleosides $\mathrm{Nu}$ cleotides 7:37-43.

Kamimura, T., Masegi, T., Urakami, K., Honda, S., Sekine, M., and Hata, T. 1983a. A new protecting tactics for the uracil residue in oligoribonucleotide synthesis. Chem. Lett. 1051-1054.

Kamimura, T., Tsuchiya, M., Koura, K., Sekine, M., and Hata, T. 1983b. Diphenylcarbamoyl and propionyl groups: A new combination of protecting groups on the guanine residue. Tetrahedron Lett. 24:2775-2778.

Khorana, H.G. 1979. Total synthesis of a gene. Science 203:614-625.

Köster, H., Kulikowski, K., Liese, T., Heikens, W., and Kohli, V. 1981. $N$-acyl protecting groups for deoxynucleosides. A quantitative and comparative study. Tetrahedron 37:363-369.
Krecmerová, M., Hrebabecky, H., and Holy, A. 1990. Synthesis of 5'-O-phosphonomethyl derivatives of pyridine 2 -deoxynucleosides. Collect. Czech. Chem. Commun. 55:2521-2536.

Kuijpers, W.H.A., Huskens, J., and van Boeckel, C.A.A. 1990. The 2-(acetoxymethyl)benzoyl (AMB) group as a new base-protecting group, designed for the protection of phosphate modified oligonucleotides. Tetrahedron Lett. 31:6729-6732.

Kume, A., Sekine, M., and Hata, T. 1982. Phthaloyl group: A new amino protecting group of deoxyadenosine in oligonucleotide synthesis. Tetrahedron Lett. 23:4365-4368.

Kume, A., Iwase, R., Sekine, M., and Hata, T. 1984. Cyclic diacyl groups for protection of the $\mathrm{N}^{6}$ amino group of deoxyadenosine in oligodeoxynucleotide synthesis. Nucl. Acids Res. 12:8525-8538.

Letsinger, R.L. and Ogilvie, K.K. 1969. Synthesis of oligothymidylates via phosphotriester intermediates. J. Am. Chem. Soc. 91:3350-3355.

Letsinger, R.L. and Lunsford, W.B. 1976. Synthesis of thymidine oligonucleotides by phosphite triester intermediates. J. Am. Chem. Soc. 98:36553661 .

Li, B.F.L., Reese, C.B., and Swann, P.F. 1987. Synthesis and characterization of oligodeoxynucleotides containing 4-O-methylthymine. Biochemistry 26:1086-1093.

MacMillan, A.M. and Verdine, G.L. 1991.Engineering tethered DNA molecules by the convertible nucleoside approach. Tetrahedron 47:26032616.

Mag, M. and Engels, J.W. 1988. Synthesis and structure assignments of amide protected nucleosides and their use as phosphoramidites in deoxyoligonucleotide synthesis. Nucl. Acids Res. 16:3525-3543.

Marugg, J.E., Tromp, M., Jhurani, P., Hoyng, C.F., van der Marel, G.A., and van Boom, J.H. 1984. Synthesis of DNA fragments by the hydroxybenzotriazole phosphodiester approach. Tetrahedron 40:73-78.

Matteucci, M.D. and Caruthers, M.H. 1980. The synthesis of oligodeoxypyrimidines on a polymer support. Tetrahedron Lett. 21:719-722.

McBride, L.J., Kierzek, R., Beaucage, S.L., and Caruthers, M.H. 1986. Amidine protecting groups for oligonucleotide synthesis. J. Am. Chem. Soc. 108:2040-2048.

McBride, L.J., Eadie, J.S., Efcavitch, J.W., and Andrus, A. 1987. Base modification and the phosphoramidite approach. Nucleosides Nucleotides 6:297-300.

Michelson, A.M. and Todd, A.R. 1955. Nucleotides. Part XXXII. Synthesis of a dithymidine dinucleotide containing a $3^{\prime}: 5^{\prime}$-internucleotidic linkage. J. Chem. Soc. 2632-2638.

Mishra, R.K. and Misra, K. 1986. Improved synthesis of oligodeoxyribonucleotide using 3methoxy-4-phenoxybenzoyl group for amino protection. Nucl. Acids Res. 14:6197-6213.
Protection of Nucleosides for Oligonucleotide Synthesis 
Mullah, B., Andrus, A., Zhao, H., and Jones, R.A. 1995. Oxidative conversion of $N$-dimethylformamidine nucleosides to $N$-cyano nucleosides. Tetrahedron Lett. 36:4373-4376.

Nagaich, A.K. and Misra, K. 1989. Highly efficient synthesis of oligodeoxyribonucleotides using $\alpha$ phenyl cinnamoyl group for selective amino protection. Nucl. Acids Res. 17:5125-5134.

Narang, S.A., Itakura, K., and Wightman, R.H. 1972. A simplification in the synthesis of deoxyribooligonucleotides. Can. J. Chem. 50:769770.

Nielsen, J., Dahl, O., Remaud, G., and Chattopadhyaya, J. 1987. Phosphitylation of guanine or inosine bases during the preparation of nucleoside phosphoramidites. Isolation of model products as thiophosphoric amide derivatives and structure elucidation by ${ }^{15} \mathrm{~N}$ NMR spectroscopy. Acta. Chem. Scand. B41:633-639.

Nyilas, A., Zhou, X.-X., Welch, C.J., and Chattopadhyaya, J. 1987. A versatile strategy for the O4protection and modification of the lactam function of uridine and uridylic acid. Nucl. Acids Res. Symp. Ser. 18:157-160.

Nyilas, A., Földesi, A., and Chattopadhyaya, J. 1988. Arenesulfonylethoxycarbonyl-A set of amino protecting groups for DNA and RNA synthesis. Nucleosides Nucleotides 7:787-793.

Ogilvie, K.K., Nemer, M.J., Hakimelahi, G.H., Proba, Z.A., and Lucas, M. 1982. N-Levulination of nucleosides. Tetrahedron Lett. 23:26152618.

Oivanen, M., Lönnberg, H., Zhou, X.X., and Chattopadhyaya, J. 1987. Acidic hydrolysis of 6-substituted 9-(2-deoxy- $\beta$-D-erythro-pentofuranosyl)purines and their 9-(1-alkoxyethyl) counterparts: Kinetics and mechanism. Tetrahedron 43:1133-1140.

Paul, C.H. and Royappa, A.T. 1996. Acid binding and detritylation during oligonucleotide synthesis. Nucl. Acids Res. 24:3048-3052.

Pfister, M. and Pfleiderer, W. 1989. New results in oligoribonucleotide synthesis. Nucleosides $\mathrm{Nu}$ cleotides 8:1001-1006.

Pfister, M., Farkas, S., Charubala, R., and Pfleiderer, W. 1988. Recent progress in oligoribonucleotide synthesis. Nucleosides Nucleotides 7:595-600.

Pfleiderer, W., Himmelsbach, F., Charubala, R., Schirmeister, H., Beiter, A., Schulz, B., and Trichtinger, T. 1985. The p-nitrophenylethyl group-An universal blocking group in nucleoside and nucleotide chemistry. Nucleosides $\mathrm{Nu}$ cleotides 4:81-94.

Pon, R.T., Damha, M.J., and Ogilvie, K.K. 1985a. Necessary protection of the $\mathrm{O}^{6}$-position of guanosine during the solid phase synthesis of oligonucleotides by the phosphoramidite approach. Tetrahedron Lett. 26:2525-2528.

Pon, R.T., Damha, M.J., and Ogilvie, K.K. 1985b. Modification of guanine bases by nucleoside phosphoramidite reagents during the solid phase synthesis of oligonucleotides. Nucl. Acids Res. 13:6447-6465.
Prasad, A.K. and Wengel, J. 1996. Enzyme-mediated protecting group chemistry on the hydroxyl groups of nucleosides. Nucleosides Nucleotides 15:1347-1359.

Rao, T.S., Reese, C.B., Serafinowska, H.T., Takaku, H., and Zappia, G. 1987. Solid-phase synthesis of the 3 'terminal nonadecaribonucleoside octadecaphosphate sequence of yeast alanine transfer ribonucleic acid. Tetrahedron Lett. 28:48974900 .

Reddy, M.P., Hanna, N.B., and Farooqui, F. 1994. Fast cleavage and deprotection of oligonucleotides. Tetrahedron Lett. 35:4311-4314.

Reese, C.B. 1978. The chemical synthesis of oligoand poly-nucleotides by the phosphotriester approach. Tetrahedron 34:3143-3179.

Reese, C.B. and Ubasawa, A. 1980. Reaction between 1-arenesulphonyl-3-nitro-1,2,4-triazoles and nucleoside residues. Elucidation of the nature of side-reactions during oligonucleotide synthesis. Tetrahedron Lett. 21:2265-2268.

Reese, C.B. and Skone, P.A. 1984. The protection of thymine and guanine residues in oligodeoxyribonucleotide synthesis. J. Chem. Soc. Perkin Trans. 1:1263.

Romero, R., Stein, R., Bull, H.G., and Cordes, E.H. 1978. Secondary deuterium isotope effects for acid-catalyzed hydrolysis of inosine and adenosine. J. Am. Chem. Soc. 100:7620-7624.

Saenger, W. 1984. Principles of Nucleic Acids Structure. Springer-Verlag, New York.

Scalfi-Happ, C., Happ, E., and Chládek, S. 1987. New approach to the synthesis of $2^{\prime}\left(3^{\prime}\right)-\mathrm{O}$-aminoacyl-oligoribonucleotides related to the $3^{\prime}$-terminus of aminoacyl transfer ribonucleic acid. Nucleosides Nucleotides 6:345-348.

Schaller, H., Weimann, G., Lerch, W.B., and Khorana, H.G. 1963. Studies on polynucleotides. XXIV. The stepwise synthesis of specific deoxyribopolynucleotides (4). Protected derivatives of deoxyribonucleosides and new syntheses of deoxyribonucleoside-3' phosphates. J. Am. Chem. Soc. 85:3821-3827.

Schulhof, J.C., Molko, D., and Teoule, R. 1987. Facile removal of new base protecting groups useful in oligonucleotide synthesis. Tetrahedron Lett. 28:51-54.

Seela, F. and Driller, H. 1989. 7-Deaza-2'-deoxy$\mathrm{O}^{6}$-methylguanosine: Selective $\mathrm{N}^{2}$-formylation via a formamidine, phosphoramidite. Synthesis and properties of oligonucleotides. Nucleosides Nucleotides 8:1-21.

Sekine, M. 1989. General method for the preparation of $\mathrm{N}^{3}$ - and $\mathrm{O}^{4}$-substituted uridine derivatives by phase-transfer reactions. J. Org. Chem. 54:2321-2326.

Sekine, M., Masuda, N., and Hata, T. 1985. Introduction of the $4,4^{\prime}, 4^{\prime \prime}$-tris(benzoyloxy)trityl group into the exo amino groups of deoxyribonucleosides and its properties. Tetrahedron 41:5445-5453. 
Shabarova, Z. and Bogdanov, A. 1994. Advanced organic chemistry of nucleic acids. VCH Publishers, New York.

Shimidzu, T. and Letsinger, R.L. 1968. Synthesis of deoxyguanylyl-deoxyguanosine on an insoluble polymer support. J. Org. Chem. 33:708-711.

Singh, R.K. and Misra, K. 1988. Improvements in oligodeoxyribonucleotide synthesis using phenoxyacetyl as amino protecting group. Indian $J$. Chem. 27B:409-417.

Sinha, N.D., Davis, P., Usman, N., Pérez, J., Hodge, R., Kremsky, J., and Casale, R. 1993. Labile exocyclic amine protection of nucleosides in DNA, RNA and oligonucleotide analog synthesis facilitating N-deacylation, minimizing depurination and chain degradation. Biochimie 75:1323.

Smrt, J. and Sorm, F. 1967. Oligonucleotidic compounds. XVIII. Synthesis of guanylyl- $\left(3^{\prime}-5^{\prime}\right)$ uridylyl- $\left(3^{\prime}-5^{\prime}\right)$-arabinofuranosyluracil and guanylyl-( $\left(3^{\prime}-5^{\prime}\right)$-uridylyl-( $\left(3^{\prime}-5^{\prime}\right)$-arabinofurano -sylcytosine. Collect. Czech. Chem. Commun. 32:3169-3176.

Sonveaux, E. 1986. The organic chemistry underlying DNA synthesis. Bioorganic Chem. 14:274325 .

Sproat, B.S., Iribarren, A.M., Guimil Garcia, R., and Beijer, B. 1991. New synthetic routes to synthons suitable for 2'-O-allyloligoribonucleotide assembly. Nucl. Acids Res. 19:733-738.

Sung, W.L. 1982. Synthesis of 4-(1,2,4-triazol-1yl)pyrimidin-2 $(1 \mathrm{H})$-one-ribonucleotide and its application in synthesis of oligoribonucleotides. J. Org. Chem. 47:3623-3628.

Suzuki, T., Ohsumi, S., and Makino, K. 1994. Mechanistic studies on depurination and apurinic site chain breakage in oligodeoxyribonucletides. Nucl. Acids Res. 22:4997-5003.

Takaku, H., Imai, K., and Nagai, M. 1988. Triphenylmethanesulfenyl group. A new protecting group for the uracil residue in oligoribonucleotide synthesis. Chem. Lett. 857-860.

Tanimura, H., Fukazawa, T., Sekine, M., Hata, T., Efcavitch, J.W., and Zon, G. 1988. The practical synthesis of RNA fragments in the solid phase approach. Tetrahedron Lett. 29:577-578.

Ti, G.S., Gaffney, B.L., and Jones, R.A. 1982. Transient protection: Efficient one-flask synthesis of protected deoxynucleosides. J. Am. Chem. Soc. 104:1316-1319.

Trichtinger, T., Charubala, R., and Pfleiderer, W. 1983. Synthesis of $\mathrm{O}^{6}$-p-nitrophenylethyl guanosine and 2 -deoxyguanosine derivatives. Tetrahedron Lett. 24:711-714.

Uchiyama, M., Aso, Y., and Noyori, R. 1993. O-Selective phosphorylation of nucleosides without N-protection. J. Org. Chem. 58:373-379.
Urdea, M.S., Ku, L., Horn, T., Gee, Y.G., and Warner, B.D. 1986. Base modification and cloning efficiency of oligodeoxyribonucleotides synthesized by the phosphoramidite method: Methyl versus cyanoethyl phosphorous protection. Nucl. Acids Res. Symp. Ser. 16:257-260.

Uznanski, B., Grajkowski, A., and Wilk, A. 1989. The isopropoxyacetic group for convenient base protection during solid-support synthesis of oligodeoxyribonucleotides and their triester analogs. Nucl. Acids Res. 17:4863-4871.

Van Aerschot, A., Herdewijn, P., Janssen, G., and Vanderhaeghe, H. 1988. Protection of the lactam function of $2^{\prime}$-deoxyinosine with a 2-(4-nitrophenyl)-ethyl moiety. Nucleosides Nucleotides 7:519-536.

Vu, H., McCollum, C., Jacobson, K., Theisen, P., Vinayak, R., Spiess, E., and Andrus, A. 1990. Fast oligonucleotide deprotection phosphoramidite chemistry for DNA synthesis. Tetrahedron Lett. 31:7269-7272.

Wagner, T. and Pfleiderer, W. 1997. Aglycone protection by the (2-dansylethoxy)carbonyl $(=\{2-$ \{[5-(dimethylamino)naphthalen-1-yl] sulfonyl \}ethoxy \} carbonyl; dnseoc) group-A new variation in oligodeoxyribonucleotide synthesis. Helv. Chim. Acta 80:200-212.

Watanabe, K.A. and Fox, J.J. 1966. A simple method for selective acylation of cytidine on the 4-amino group. Angew. Chem. Intl. Ed. Engl. 5:579-580.

Welch, C.J., Bazin, H., Heikkilä, J., and Chattopadhyaya, J. 1985. Synthesis of C-5 and N-3 arenesulfenyl uridines. Preparation and properties of a new class of uracil protecting group. Acta Chem. Scand. B39:203-212.

York, J.L. 1981. Effect of structure of the aglycon on the acid-catalyzed hydrolysis of adenine nucleosides. J. Org. Chem. 46:2171-2173.

Zhou, X.-X. and Chattopadhyaya, J. 1986. Site-specific modification of the pyrimidine residue during the deprotection of the fully-protected diuridylic acid. Tetrahedron 42:5149-5156.

Zhou, X.X., Sandström, A., and Chattopadhyaya, J. 1986. A convenient preparation of $2-N-(4-t-$ butylbenzoyl)-6-O-(2-nitrophenyl)guanosine and its application in the synthesis of $5^{\prime}(\mathrm{GpGpGpU}) 3^{\prime}$ constituting the $3^{\prime}$-anticodon stem of E.coli tRNA ${ }^{\text {lle }}$. Chem. Scr. 26:241-249.

Zoltewicz, J.A. and Clark, D.F. 1972. Kinetics and mechanism of the hydrolysis of guanosine and 7-methylguanosine nucleosides in perchloric acid. J. Org. Chem. 37:1193-1197.

Zoltewicz, J.A., Clark, D.F., Sharpless, T.W., and Grahe, G. 1970. Kinetics and mechanism of the hydrolysis of some purine nucleosides. J. Am. Chem. Soc. 92:1741-1750.

Contributed by Radhakrishnan P. Iyer

OriGenix Technologies

Laval, Quebec, Canada
Protection of Nucleosides for Oligonucleotide Synthesis 